\title{
Genotipagem por spoligotyping de Mycobacterium tuberculosis obtidos de lâminas de Ziehl-Neelsen em Belém, Estado do Pará, Brasil
}

Spoligotyping of Mycobacterium tuberculosis in Ziehl-Neelsen-stained slides from Belém, Pará State, Brazil

Genotipado por spoligotyping de Mycobacterium tuberculosis obtenidos de láminas de Ziehl-Neelsen en Belém, Estado de Pará, Brasil

Ismari Perini Furlaneto

Núcleo de Medicina Tropical, Universidade Federal do Pará, Belém, Pará, Brasil

Emilyn Costa Conceição

Programa de Pós-graduação em Biologia Parasitária da Amazônia, Universidade do Estado do Pará, Belém, Pará, Brasil

Michele Lima de Brito

Núcleo de Medicina Tropical, Universidade Federal do Pará, Belém, Pará, Brasil

Ana Roberta Fusco da Costa

Seção de Bacteriologia e Micologia, Instituto Evandro Chagas/SVS/MS, Ananindeua, Pará, Brasil
João Júlio Batista Monteiro

Laboratório de Geoprocessamento/Instituto Evandro Chagas/SVS/MS, Ananindeua, Pará, Brasil

Nelson Veiga Gonçalves

Laboratório de Geoprocessamento/Instituto Evandro Chagas/SVS/MS, Ananindeua, Pará, Brasil

Harrison Magdinier Gomes

Laboratório de Biologia Molecular Aplicada a Micobactérias/ Fundação Oswaldo Cruz, Rio de Janeiro, Brasil

Karla Valéria Batista Lima

Seção de Bacteriologia e Micologia, Instituto Evandro Chagas/SVS/MS, Ananindeua, Pará, Brasil

\section{RESUMO}

O spoligotyping é uma técnica molecular baseada na amplificação de uma região de repetições diretas polimórficas, a qual permite detectar e diferenciar Mycobacterium tuberculosis (MT). Neste estudo, foram genotipados MT obtidos de material fixado em lâminas coradas pela técnica de Ziehl-Neelsen (ZN), confeccionadas em laboratórios públicos de Belém. A identificação molecular de MT por spoligotyping em amostras de 102 indivíduos apresentou elevada sensibilidade, com 90 padrões de hibridização completos e concordantes entre si. A classificação dos genótipos foi realizada comparando os resultados obtidos com aqueles disponíveis no banco de dados internacional SITVITWEB. Foram observados 45 genótipos distintos, dos quais 35 previamente relatados e 11 ainda não relatados. Sessenta e um genótipos apresentaram-se compartilhados por duas a 15 amostras e 29 genótipos eram únicos. As famílias LAM, T, H e EAl foram as mais frequentes. Os bairros com maior concentração de casos foram Guamá, Jurunas e Terra Firme. A genotipagem por spoligotyping torna-se uma importante ferramenta no monitoramento de isolados em diferentes contextos epidemiológicos. A possibilidade de caracterizar genética e demograficamente estes microorganismos contribui para o melhor entendimento de como a doença é distribuída nesta população, no esclarecimento das transmissões e das contaminações cruzadas e na implementação de ações para o controle da tuberculose.

Palavras-chave: Mycobacterium tuberculosis; Tuberculose; Spoligotyping; Patologia Molecular; Técnicas de Tipagem Bacteriana; EAl.

\section{INTRODUÇÃO}

No ano de 2010 foram notificados, no Brasil, 71 mil casos novos de tuberculose (TB), de todas as formas, ocorrendo 4,6 mil mortes devido à doença,

\footnotetext{
Correspondência / Correspondence / Correspondencia:

Karla Valéria Batista Lima

Instituto Evandro Chagas, Seção de Bacteriologia e Micologia

Rodovia BR 316, km7, s/nº. Bairro: Levilândia

CEP: 67030-000 Ananindeua-Pará-Brasil

E-mail: karlavaleria_2007@hotmail.com
}

correspondendo a uma incidência de 37,7/100 mil habitantes e a uma taxa de mortalidade estimada em 2,4/100 mil habitantes'. O Pará é o Estado da Região Norte que concentra o maior número de casos novos da doença, tendo respondido por $50 \%$ das notificações em 2011 , com 3,364 casos novos e uma taxa de incidência estimada em 47,3/100 mil habitantes, a terceira maior entre os estados brasileiros ${ }^{2}$.

O Pará abriga 11 das 315 cidades que foram definidas pelo Ministério da Saúde (MS) como prioritárias para o controle da TB no Brasil porque, juntas, concentram 75\% dos casos da doença no país. Belém, a sua capital, e 
outras dez cidades somam $60 \%$ dos casos no Pará ${ }^{\text {. A }}$ caracterização genética e demográfica de MT contribui para elucidar alguns aspectos quanto à dispersão deste micro-organismo, além de possibilitar a implementação de ações para seu controle e combate.

A genotipagem do MT tem sido um bom instrumento no estudo das relações de clonalidade e dispersão de cepas em grupos populacionais ${ }^{4}$. Nos últimos anos, foram desenvolvidos métodos de tipagem baseados na reação em cadeia da polimerase $(P C R)^{4,7}$ que têm como vantagens a utilização de baixa quantidade de DNA como alvo para tipagem e serem de fácil execução. Destes, por sua simplicidade e demais vantagens, como menor custo e uso de espécimes clínicos diversos, o spoligotyping é atualmente o mais utilizado, também como método de tipagem secundário para cepas com menos de cinco cópias da IS61108,12.

Embora o RFLP-IS6110 seja considerado o método padrão-ouro para tipagem de membros do complexo M. tuberculosis (CMTB) diversas desvantagens têm sido relatadas quanto ao seu uso ${ }^{7}$. A técnica de spoligotyping tem sido empregada com sucesso em diversos tipos de amostras, tais como tecidos preservados em parafina, tecidos mumificados, amostras de escarro e lâminas coradas pela técnica de $Z \mathrm{~N}$, tanto recentes quanto antigas $^{13,15}$.

Neste estudo, foram genotipados, por spoligotyping, MT obtidos a partir de lâminas coradas pela técnica de $Z N$, confeccionadas em unidades laboratoriais (UL) da rede pública de Belém entre outubro de 2007 e março de 2008, avaliando-se a aplicabilidade da técnica neste tipo de amostra e a distribuição dos genótipos na região.

\section{MATERIAIS E MÉTODOS}

estudo foi dividido em duas fases: na primeira, foram selecionadas, aleatoriamente, 102 lâminas com baciloscopia positiva e com diferentes contagens de bacilos álcool-ácido resistentes (BAAR); na segunda, foram selecionados 32 pacientes, dentre os 102, que realizaram o exame mais de uma vez e tiveram, individualmente, uma lâmina extra incluída no estudo, a qual foi utilizada para comparar os perfis de hibridização obtidos em diferentes amostras de um mesmo indivíduo. Desta forma, as amostras clínicas consistiram em 134 lâminas com esfregaços de escarro previamente coradas pela técnica de ZN, provenientes de algumas UL, encaminhadas ao Laboratório Central do Pará (LACEN-PA) e cedidas para o desenvolvimento deste trabalho.

\section{SPOLIGOTYPING}

O DNA foi extraído conforme proposto por Furlaneto et $\mathrm{al}^{12}$. $\bigcirc$ spoligotyping foi realizado de acordo com as instruções do fabricante (Isogen Bioscience B.V., Maarsen, HOLANDA), com algumas modificações ${ }^{16}$. Para verificar a reprodutibilidade da técnica, o spoligotyping foi realizado em duplicata a partir de nova amplificação do DNA das mesmas amostras para ambas as fases em dias distintos e utilizando-se membranas diferentes. O MT H37Rv e M. bovis foram utilizados como controle positivo. Como controle negativo utilizou-se água previamente submetida aos procedimentos de extração. Este controle negativo também foi usado para avaliar a possibilidade de contaminação cruzada laboratorial.

Os resultados foram analisados visualmente por dois avaliadores experientes, para estabelecer um consenso de leitura dos padrões. Os genótipos obtidos foram inscritos em formato binário como planilhas de Excel ${ }^{\circledR}$ (Microsoft, Redmond, WA, EUA) e comparados com os padrões descritos no banco de dados SITVIT (disponível em: http://www.pasteur-guadeloupe.fr:8081/SITVITDemo/), a fim de identificar o Spoligo Internacional Type (SIT) correspondente. Padrões ainda não descritos foram classificados em prováveis famílias de acordo com Vitol et al $^{17}$ (disponível em: http://cgi2.cs.rpi.edu/ bennek/ SPOTCLUST.html). Os genótipos que não parearam com nenhum outro previamente descrito nos bancos de dados foram definidos neste estudo como órfãos. Foi definido como cluster um grupo de duas ou mais amostras que tiveram padrões idênticos de spoligotyping provenientes de indivíduos distintos.

\section{GEOLOCALIZAÇÃO DOS CASOS}

A localização dos casos incluídos no estudo foi analisada através do registro de coordenadas geográficas (latitude e longitude) no local de residência de cada paciente, com o auxílio de aparelho receptor do Sistema de Posicionamento Global (GPS) (GPS Map60CSx, GARMIN, Olathe, Kansas, EUA), e, para a base de dados formada com estas informações, foi utilizado software compatível para integrar as bases cartográficas, imagens digitais e o conteúdo do estudo (ArcGIS 9.2, ESRI, EUA; TerraView 3.1, INPE, Brasil). A base cartográfica do Município de Belém foi cedida pelo Instituto Brasileiro de Geografia e Estatística (IBGE) para o Laboratório de Geoprocessamento do Instituto Evandro Chagas.

endereço dos indivíduos foi obtido por meio de consulta ao Livro de Baciloscopia e Cultura enviado pela unidade laboratorial (UL) ao LACEN-Pa.

\section{ANÁLISE ESTATÍSTICA}

As análises estatísticas foram realizadas utilizando o BioEstat, versão $5.0^{18}$. Foram utilizados os testes $\chi 2 \mathrm{e}$ Kappa e o valor de $\mathrm{p} \leq 0.05$ foi usado para definir a significância das variáveis.

\section{APROVAÇÃO ÉTICA}

$\bigcirc$ projeto foi aprovado pelo Comitê de Ética em Pesquisa com Humanos do Instituto Evandro Chagas (CEP/IEC/SVS/MS) em 06 de abril de 2009, parecer $n^{\circ}$ 003/2009, protocolo CEP/IEC n 0004/2009, CAAE: 0004.0.072.073-09.

\section{RESULTADOS}

Em 93/102 DNA (91,2\%) obtidos de esfregaço de escarro de pacientes com bacterioscopia positiva para BAAR, o spoligotyping foi bem sucedido, apresentando padrões genéticos reprodutíveis nos DNA de lâminas em duplicata dos mesmos pacientes. Apenas 8,8\% das amostras tiveram padrões duvidosos, não sendo possível elucidá-los mesmo após repetição da genotipagem. Todas as amostras que apresentaram padrões duvidosos 
permaneceram sem definição mesmo após a repetição do experimento. Os controles positivos MT H37Rv e M. bovis apresentaram os padrões de hibridização característicos e não houve sinal de hibridização entre os controles negativos. Não foi significativa a diferença quanto à carga bacilar entre as amostras que apresentaram padrões de hibridização completos e as de padrão duvidoso ( $p=0,2335)$.

Em três casos foram observadas discordâncias entre os perfis de hibridização apresentados por amostras diferentes de um mesmo indivíduo, embora todas apresentassem resultados concordantes entre si após as duplicatas.

Das 90 amostras com padrão genético completo e sem discordância, 79 (87,8\%) foram classificadas em 35 SIT. Destas, 20 apresentaram padrões únicos e 15, padrões compartilhados (Tabela 1). Onze amostras exibiram padrões genéticos ainda não descritos, compreendendo nove padrões únicos e um padrão compartilhado por duas amostras (Tabela 2). Entre estes genótipos, três pareavam com perfis descritos como órfãos no SITVITWEB, sendo estes provenientes do Brasil, Itália e Portugal.

Tabela 1 - Frequência dos espoligotipos encontrados entre as amostras de Belém, identificados com um número SIT segundo o banco de dados internacional SITVITWEB ${ }^{19}$.

\begin{tabular}{|c|c|c|c|c|c|}
\hline SIT & ID & $\mathrm{n}$ & Freq (\%) & Espoligopadrão Espaçadores 1-43 & Octal \\
\hline 53 & $\mathrm{Tl}$ & 15 & 18,9 & 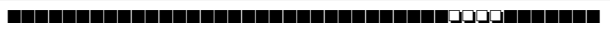 & 777777777760771 \\
\hline 42 & LAM9 & 10 & 12,7 & 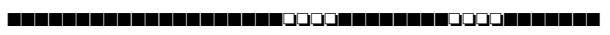 & 777777607760771 \\
\hline 100 & Manul & 4 & 5,1 & 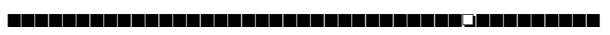 & 777777777773771 \\
\hline 93 & LAM5 & 4 & 5,1 & 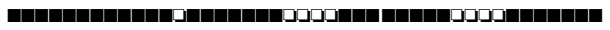 & 777737607760771 \\
\hline 137 & X2 & 3 & 3,8 & 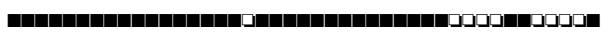 & 777776777760601 \\
\hline 17 & LAM2 & 3 & 3,8 & 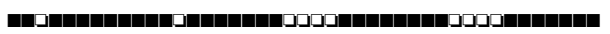 & 677737607760771 \\
\hline 50 & H3 & 3 & 3,8 & 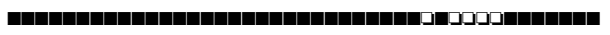 & 777777777720771 \\
\hline 740 & $\mathrm{H} 3$ & 3 & 3,8 & 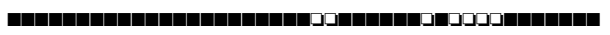 & 777777747720771 \\
\hline 1580 & $\mathrm{~T}$ & 2 & 2,5 & 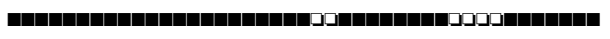 & 777777747760771 \\
\hline 1690 & Manu2 & 2 & 2,5 & | | & 777777777762771 \\
\hline 64 & LAM6 & 2 & 2,5 & 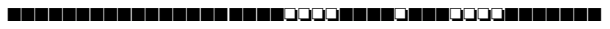 & 777777607560771 \\
\hline 33 & LAM3 & 2 & 2,5 & 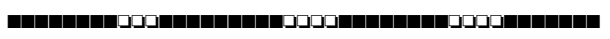 & 776177607760771 \\
\hline 20 & LAM 1 & 2 & 2,5 & 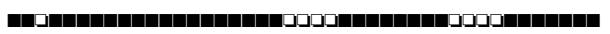 & 677777607760771 \\
\hline 129 & EAI6-BGDI & 2 & 2,5 & 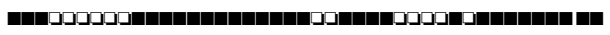 & 700777747413771 \\
\hline 48 & EAII-SOM & 2 & 2,5 & 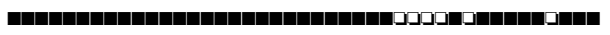 & 777777777413731 \\
\hline 4 & Unknown & 1 & 1,3 & 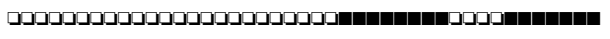 & 000000007760771 \\
\hline 46 & Unknown & 1 & 1,3 & 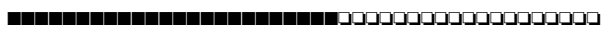 & 777777770000000 \\
\hline 60 & LAM4 & 1 & 1,3 & 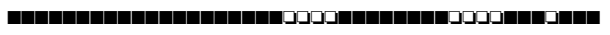 & 777777607760731 \\
\hline 73 & $\mathrm{~T}$ & 1 & 1,3 & 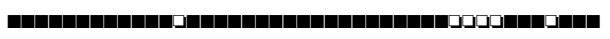 & 777737777760731 \\
\hline 92 & X3 & 1 & 1,3 & 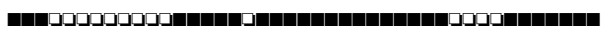 & 700076777760771 \\
\hline 95 & LAM6 & 1 & 1,3 & 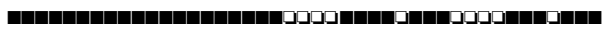 & 777777607560731 \\
\hline 177 & LAM9 & 1 & 1,3 & 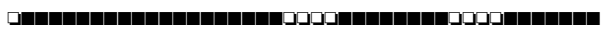 & 377777607760771 \\
\hline 209 & LAM12-Madrid 1 & 1 & 1,3 & 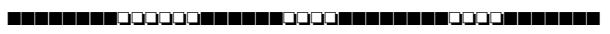 & 776017607760771 \\
\hline 216 & LAM5 & 1 & 1,3 & 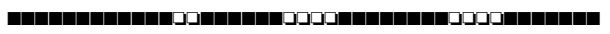 & 777717607760771 \\
\hline 244 & T1 & 1 & 1,3 & 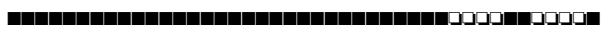 & 777777777760601 \\
\hline 245 & $\mathrm{~T} 1$ & 1 & 1,3 & 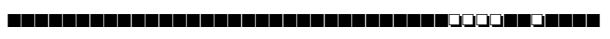 & 777777777760671 \\
\hline 373 & $\mathrm{Tl}$ & 1 & 1,3 & 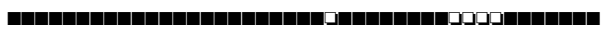 & 777777767760771 \\
\hline 430 & T4-CEU1 & 1 & 1,3 & 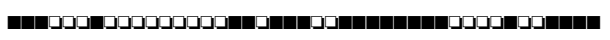 & 704003347760471 \\
\hline 435 & LAM9 & 1 & 1,3 & 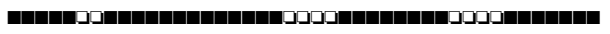 & 763777607760771 \\
\hline 763 & EAI5 & 1 & 1,3 & 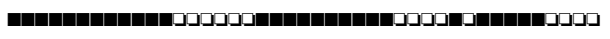 & 777700777413700 \\
\hline 888 & T2 & 1 & 1,3 & 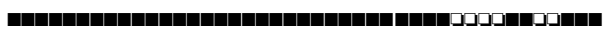 & 777777777760631 \\
\hline 1076 & LAM1 & 1 & 1,3 & 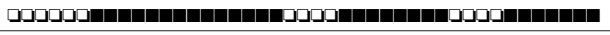 & 007777607760771 \\
\hline
\end{tabular}

SITVITWEB: Banco Mundial de Espoligotipos; SIT: Spoligo-International-Type number; ID: Identificação; n: Número de amostras; Freq: Frequência; EAl: East African-Indian; LAM: Latin American and Mediterranean; T e X: Famílias "T" e "X", respectivamente; MAD: Madri; CEU: Central Europe; U: Unknown (desconhecida).

Tabela 2 - Frequência de espoligopadrões (agrupados e únicos) sem um número SIT descrito no SITVITWEB, encontrados entre as amostras de Belém, e classificados de acordo com as regras propostas por Vitol et al ${ }^{17}$.

\begin{tabular}{|c|c|c|c|c|c|c|c|c|}
\hline ID* & $\begin{array}{l}\text { ID } \\
\text { SpotClust }\end{array}$ & $\begin{array}{l}\text { Probab } \\
\text { SpotClust }\end{array}$ & $n$ & $\begin{array}{l}\text { Freq } \\
(\%)\end{array}$ & $\begin{array}{c}\text { Espoligopadrão } \\
\text { Espaçadores 1- } 43\end{array}$ & Octal & FAM/SUBF & SIT \\
\hline VO8 & $\mathrm{Tl}$ & 0,99 & 2 & 2,3 & & 777737777760071 & Orphan & Orphan \\
\hline $\mathrm{PO}^{\mathrm{a}}$ & LAM9 & 0.99 & 1 & 1,1 & & 777767607560671 & Orphan & Orphan \\
\hline $\mathrm{PO} 2^{\mathrm{b}}$ & $\mathrm{T} 2$ & 0.97 & 1 & 1,1 & & 737777777760700 & Orphan & Orphan \\
\hline $\mathrm{PO}^{\mathrm{C}}$ & LAMI & 0.66 & 1 & 1,1 & & 647777607760771 & Orphan & Orphan \\
\hline VO2 & EAI5 & 0,99 & 1 & 1,1 & & 777307777413621 & Orphan & Orphan \\
\hline VO3 & Haarlem3 & 0,77 & 1 & 1,1 & $\square \square$ & 777773747720771 & Orphan & Orphan \\
\hline VO4 & EAI5 & 0,99 & 1 & 1,1 & & 770355747413771 & Orphan & Orphan \\
\hline VO5 & F33 & 0,99 & 1 & 1,1 & & 17777777777327 & Orphan & Orphan \\
\hline VO6 & EAl2 & 0,68 & 1 & 1,1 & & 777600005413371 & Orphan & Orphan \\
\hline VO7 & LAM9 & 0,99 & 1 & 1,1 & | & 777307607760661 & Orphan & Orphan \\
\hline \multicolumn{3}{|c|}{ Total de amostras } & 12 & 13,3 & & & & \\
\hline
\end{tabular}

*Designação da amostra no estudo; SITVITWEB: Banco Mundial de Espoligotipos; SIT: Spoligo-International-Type number; n: número de amostras; Freq: frequência; EAI: East African-Indian; F33: Família 33; LAM: Latin American and Mediterranean; T e X: famílias "T" e "X", respectivamente; VO: verdadeiro órfão; PO: espoligotipos pseudo-órfãos: ${ }^{a, b, c}$ origem dos perfis pareados: Brasil, Itália e Portugal, respectivamente.

A família Latin American and Mediterranean (LAM) foi a mais frequente, agrupando 35 (38,8\%) amostras, seguida pelas famílias T, East African-Indian (EAl) e Haarlem (H), que apresentaram 25, 8 e 7 amostras, respectivamente. Oito SIT acomodaram $49,4 \%$ das amostras (SIT53, SIT42, SIT100, SIT93, SIT137, SIT17, SIT50 e SIT740). A 
subfamília $T 1$ reuniu 23,3\% das amostras, sendo a mais frequente neste estudo (Tabelas 1 e 2).

Dos 102 casos de Belém, foram registradas as coordenadas geográficas de $81 \quad(79,4 \%)$ deles, não sendo possível localizar o endereço dos demais (21; 20,6\%), apesar de constarem no Livro de maneira aparentemente completa. A distribuição total dos casos georreferenciados em Belém de acordo com o spoligotyping está representada na figura 1. Os 81 casos estavam distribuídos por 29 dos 71 bairros da capital. Os bairros que mais concentraram casos foram Guamá (9; $11,1 \%)$, Jurunas $(6 ; 7,4 \%)$ e Terra Firme $(5 ; 6,2 \%)$.

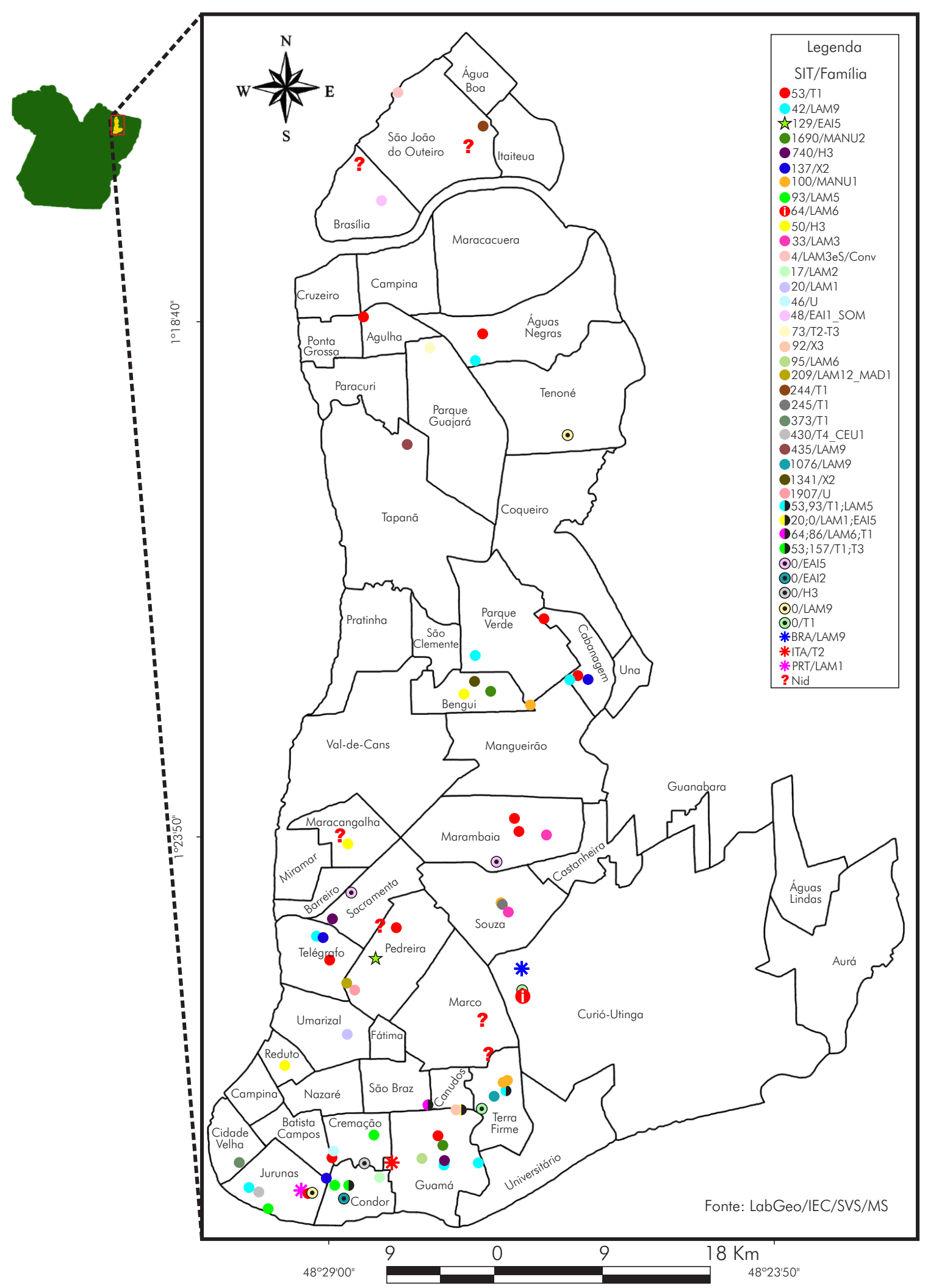

Nota: Cada círculo representa um caso e cada cor está associada a um perfil de spoligotyping, conforme legenda.

Figura 1 - Mapa ilustrando a localização geográfica, por endereço de residência, dos 81 casos geolocalizados no Município de Belém/PA. 


\section{DISCUSSÃO}

conhecimento dos genótipos circulantes em determinada área geográfica fornece informações sobre a dinâmica de transmissão do MT e, dessa forma, contribui para elaboração de estratégias para o controle da TB. Além disso, o ideal é que possam ser aplicados diretamente em espécimes clínicos, aumentando assim a oferta de amostras e diminuindo o tempo de resposta em áreas endêmicas. Recentes avanços no campo da biologia molecular têm proporcionado o desenvolvimento de técnicas que permitem a rápida identificação e o rastreamento de cepas específicas de MT que circulam no mundo. A maioria destas técnicas explora o polimorfismo presente no genoma micobacteriano, utilizando vários elementos repetitivos do DNA como marcadores moleculares genéticos, e gera perfis genéticos estirpeespecíficos.

No presente estudo, que utilizou DNA extraído de esfregaços fixados em lâminas de $Z \mathrm{~N}$, padrões genéticos completos e reprodutíveis foram obtidos em 90\% das amostras. Estudos prévios relataram rendimento menor quando a técnica foi aplicada na tipagem de material extraído de lâminas de $Z^{1} N^{14,15}$.

As famílias mais frequentes neste estudo (LAM, T e Haarlem) também reuniram maior número de casos em outros estudos realizados no Brasil20,22, assim como em outros países da América Latina ${ }^{19,23,24}$. A prevalência da família LAM, que agrupou 39,3\% das amostras genotipadas, também tem sido observada no Paraguai ${ }^{25}$ e na Venezuela ${ }^{26}$.

Amplamente distribuídos, os tipos SIT53 (T1) e SIT42 (LAM9) agruparam maior número de amostras, englobando, juntos, $27,7 \%$ de todos os casos deste trabalho. Investigações mais detalhadas devem ser realizadas para se verificar a transmissão e/ou melhor adaptação destes genótipos. Eles ocuparam, respectivamente, a $2^{a}$ e a $5^{a}$ posição entre os mais frequentes no SpolDB427. Entre os SIT encontrados, alguns ainda não foram descritos em Belém (SIT 4, 60, 216, 435 e 1907) e um ainda não foi referenciado no Brasil (SIT209), ocorrendo, porém, em países vizinhos ou com relações com o país, como Venezuela, EUA, Itália, Espanha ${ }^{24}$. O SIT1999 apresenta cinco amostras depositadas no SITVITWEB, sendo três da Guiana Francesa e duas da Itália.

Quanto à família EAI, sua presença na América do Sul é pouco relatada. De fato, nenhum isolado desta família foi relatado em diversos estudos realizados recentemente no Paraguai ${ }^{25}$, na Venezuela ${ }^{26,28}$ e no Chile $^{29}$. No entanto, há relatos na Guiana Francesa ${ }^{24}$ e no Brasil ${ }^{21}$, onde observaram-se particularidades em relação à distribuição desta família no país. Gomes et $a^{21}$, em estudos realizados em 11 Estados brasileiros, descrevem a presença de cepas de EAI somente no Pará, Pernambuco e Rio de Janeiro, sendo que 76,2\% dos isolados eram provenientes do Pará, onde estavam presentes os SIT 48 e 129, os quais foram também descritos no presente trabalho. Estes achados parecem confirmar a hipótese levantada por Gomes et al ${ }^{21}$, de que estas cepas foram exportadas da Guiana Francesa para
- Brasil, iá que esta família tem baixa representatividade no Brasil e é intensa na Guiana. Não foram observados genótipos pertencentes às famílias Beijing e CAS e nem de tipos pertencentes às outras espécies do CMTB, como o M. bovis, por exemplo. De fato, a presença do genótipo Beijing é raramente observada em pacientes com TB na América do Sul23,30,31.

As três amostras que apresentaram perfis de spoligotyping discordantes foram excluídas das comparações com os bancos de dados. No entanto, o fato de seus resultados terem se confirmado após as duplicatas, aliado à ausência de hibridização entre os controles negativos, pode sugerir a presença de genótipos diferentes em um mesmo paciente. Embora tradicionalmente seja assumido que a TB ativa é resultado de infecção com único clone bacteriano e que recorrências são resultado da reativação da cepa que causou o primeiro episódio, infecções simultâneas por múltiplas cepas ou reinfecção por uma segunda estirpe de MT podem ser responsáveis por uma parcela dos casos da doença ${ }^{32-36}$. No entanto, considera-se importante utilizar reagentes que evitem o carreamento de DNA e posterior contaminação, como uracil DNA glicosilase e dUTP ${ }^{37}$. Para confirmação da presença de infecção policlonal, seria necessário o cultivo micobacteriano e análise de colônias individuais por spoligotyping e uma técnica secundária, como o MIRUVNTR ou por RFLP-IS6110.

Os mapas temáticos são instrumentos valiosos na análise espacial do risco de determinada doença, permitindo sugerir a distribuição espacial da doença na região de interesse, os determinantes locais, fatores etiológicos e apontar associações entre as fontes de contaminação e as áreas de risco elevado ${ }^{38}$.

A proposta do presente estudo, em relação à distribuição espacial dos casos, foi descritiva. Por meio da localização pontual da residência dos casos e ilustração dos mesmos em mapas, foi possível observar áreas onde houve certo agrupamento de casos com o mesmo perfil de Spoligotyping, como aconteceu com os casos pertencentes aos agrupamentos SIT64 (mesma residência), SIT100 (100 m) e SIT53 (290 m), em Belém. Este fato chama atenção para a possibilidade de que os casos que possuem o mesmo genótipo participem da mesma cadeia de transmissão, ressaltando a importância de aumentar a vigilância e investigação nesta área. Por outro lado, como o período selecionado para obtenção das amostras foi de seis meses e não envolveu a totalidade dos casos com baciloscopia positiva, algumas áreas não tiveram nenhum ponto localizado (como foi o caso da parte leste de Belém), o que não significa ausência de casos nestas localidades.

Alguns bairros apresentaram maior concentração de pontos (casos), independentemente do genótipo (SIT): os bairros Guamá, Jurunas e Terra Firme, que, em conjunto, concentraram 24,7\% dos casos geolocalizados. Avaliando as características da população neles residente, observase que estão entre os bairros mais populosos da capital paraense e contam com as médias mais elevadas de moradores por domicílio 39,40 . 
Outro fator que influenciou na redução dos casos disponíveis para este estudo foi a exclusão inicial de $27,2 \%$ das amostras por motivos relacionados ao endereço (ausente/ incompleto/ outro município). Em estudo exploratório realizado por Dos Santos et $a^{41}$, frente à dificuldade de localizar alguns endereços, os autores presumiram que os endereços incompletos e consequentemente difíceis de serem localizados pertenciam a áreas carentes, podendo resultar em uma subestimação de casos nos bolsões urbanos de pobreza.

De qualquer maneira, estes fatores não tiveram tanta influência no resultado final deste estudo, já que ele não teve finalidade exploratória ou ecológica e tampouco de ilustrar a incidência dos casos de TB. Além disso, pela própria característica de uma das UL selecionadas (HUJBB), que produz muitos exames, porém atende grande número de pacientes de outros municípios, a amostra foi sendo reduzida, o que se deveu também pela dificuldade de localizar os endereços, pois a única fonte de dados era a cópia do Livro de Baciloscopia e Cultura enviada pelas UL. Umas das dificuldades em se trabalhar com dados secundários é o fato de que, em muitas situações, o registro incompleto - ou até mesmo a falta de registro - das informações impossibilita uma abordagem ampla e fiel dos achados. Neste estudo, este fato foi evidenciado principalmente em relação aos endereços: muitos não constavam nos registros que acompanhavam as amostras ou, quando constavam, não continham os elementos básicos (rua, número, bairro), fato que tem sido notado em diversos estudos ${ }^{42}$.

Estudos que avaliam a totalidade de isolados disponíveis de uma determinada área geográfica em um tempo definido podem ajudar a estimar a importância da transmissão recente e a identificar os fatores de risco para a disseminação da TB ${ }^{43}$. É importante salientar que investigações de traçado convencional de contatos identificam somente uma pequena parcela dos casos envolvidos em uma cadeia de transmissão, o que exige novas abordagens ${ }^{44}$.

\section{CONCLUSÃO}

A técnica spoligotyping é simples, prática e reprodutiva para genotipagem de MT provenientes de lâminas coradas pelo método de Ziehl-Neelsen, permitindo inclusive a detecção de infecções mistas, podendo ser inserida na rotina dos Laboratórios de Vigilância.

As famílias LAM, T e $\mathrm{H}$ foram as mais frequentes neste estudo, da mesma forma como em outros estudos realizados no Brasil e América Latina. A família EAI está entre as mais frequentes na população estudada; no entanto, é raramente relatada na América Latina.

$\bigcirc$ preenchimento inadequado das informações referentes ao endereço dos indivíduos aponta para a necessidade de qualificar o sistema de coleta, organização e armazenamento de dados, para que estes possam ser acessados quando necessário e, validados, se tornem parte integrante da vigilância da TB e de outras doenças que possam ser objeto de investigação futura. Somente desta forma estes dados permitirão a utilização de abordagens de estatística espacial que ajudem a definir, de forma clara e segura, os fatores de risco aos quais a comunidade está exposta.

\section{AGRADECIMENTOS}

À farmacêutica Zelinda Habib Dantas de Santana, responsável pelo Serviço de Tuberculose/ LACEN-PA e ao farmacêutico Kleiffson Alves de Miranda, diretor do LACEN-PA por autorizar o fornecimento das lâminas.

\section{APOIO FINANCEIRO}

Este trabalho foi realizado com apoio financeiro do Instituto Evandro Chagas/ SVS/ MS, Coordenação de Aperfeiçoamento de Pessoal de Nível Superior e Programa de Iniciação Científica/ IEC/ CNPq.

\title{
Spoligotyping of Mycobacterium tuberculosis in Ziehl-Neelsen-stained slides from Belém, Pará State, Brazil.
}

\begin{abstract}
Spoligotyping is a molecular method based on the amplification of a polymorphic direct repeat locus, which allows for the detection and identification of Mycobacterium tuberculosis. Samples of M. tuberculosis obtained from material fixed in Ziehl-Neelsen-stained slides from public laboratories in Belém, Pará State, Brazil, were genotyped in this study. The molecular characterization of M. tuberculosis through spoligotyping of samples from 102 patients showed high sensitivity, with 90 complete and matching hybridization patterns. The genotypes were classified based on a comparison of the results obtained with data available in the SITVITWEB international database. Of the 45 different genotypes observed, 35 had already been reported and 11 had never been reported. Sixty-one genotypes were observed in 2 to 15 samples, and 29 genotypes did not match. LAM, T, H and EAl were the most common families. The districts with the highest concentration of cases were Guamá, Jurunas and Terra Firme. Spoligotyping is considered an important tool to follow up isolates in distinct epidemiologic contexts. Genetic and demographic characterization of these microorganisms contributes to a better understanding of the distribution of tuberculosis in this population, its transmission process, the occurrence of cross contaminations, and the implementation of controlling measures of the disease.
\end{abstract}

Keywords: Mycobacterium tuberculosis; Tuberculosis; Spoligotyping; Pathology, Molecular; Bacterial Typing Techniques; EAl. 


\section{Genotipado por spoligotyping de Mycobacterium tuberculosis obtenidos de láminas de Ziehl-Neelsen en Belém, Estado de Pará, Brasil.}

\section{RESUMEN}

El spoligotyping es una técnica molecular basada en la amplificación de una región de repeticiones directas polimórficas, que permite detectar y diferenciar Mycobacterium tuberculosis. En este estudio, se genotiparon M. tuberculosis obtenidos de material fijado en láminas teñidas por la técnica de Ziehl-Neelsen (ZN), confeccionadas en laboratorios públicos de Belém. La identificación molecular de M. tuberculosis por spoligotyping en muestras de 102 individuos presentó una elevada sensibilidad, con 90 estándares de hibridación completos y concordantes entre sí. La clasificación de los genotipos se hizo comparando los resultados obtenidos con los disponibles en el banco de datos internacional SITVITWEB. Se observaron 45 genotipos distintos, de los cuales 35 previamente relatados y 11 todavía no relatados. Sesenta y un genotipos se presentaron compartidos en dos a 15 muestras y 29 genotipos eran únicos. Las familias LAM, T, H y EAl fueron las más frecuentes. Los barrios con mayor concentración de casos fueron Guamá, Jurunas y Terra Firme. El genotipado por spoligotyping se torna una importante herramienta en el monitoreo de aislados en diferentes contextos epidemiológicos. La posibilidad de caracterizar genética y demográficamente estos microorganismos contribuye a la mejor comprensión de como se distribuye la enfermedad en esta población, al esclarecimiento de las transmisiones y de las contaminaciones cruzadas y a la implementación de acciones para el control de la tuberculosis.

Palabras clave: Mycobacterium tuberculosis; Tuberculosis; Spoligotyping; Patología Molecular; Técnicas de Tipificación Bacteriana; EAI.

\section{REFERÊNCIAS}

1 World Health Organization. Report 2011. Global Tuberculosis Control [Internet]. 2011 [cited 2012 Jan 05]. Available from: http://www.who.int/tb/ publications/global_report/en/

2 Ministério da Saúde (BR). Secretaria de Vigilância em Saúde. Departamento de Vigilância Epidemiológica. Sistema de Informação de Agravos de Notificação SINAN/SVS/MS. Taxa de incidência de tuberculose no Brasil [Internet]. Brasília: Ministério da Saúde; 2012 [citado 2012 mar 01]. Disponível em: http://portal. saude.gov.br/portal/arquivos/pdf/taxa_incidencia tb_1990_2011_16_02_2012_pub.pdf

3 Ministério da Saúde (BR). Departamento de Vigilância das Doenças Transmissíveis. Programa Nacional de Controle da Tuberculose. Nota técnica $n^{\circ} 15$ de 7 de outubro de 2011. Municípios prioritários para controle da tuberculose. Brasília: Ministério da Saúde; 2011.

4 van Soolingen D. Molecular epidemiology of tuberculosis and other mycobacterial infections: main methodologies and achievements. J Intern Med. 2001 Jan;249(1):1-26.

5 Friedman CR, Stoeckle MY, Johnson WD, Riley LW. Double-repetitive-element PCR method for subtyping M. tuberculosis clinical isolates. J Clin Microbiol. 1995 May;33(5):1383-4.

6 Kamerbeek J, Schouls L, Kolk A, van Agterveld M, van Soolingen D, Kuijper $S$, et al. Simultaneous detection and strain differentiation of Mycobacterium tuberculosis for diagnosis and epidemiology. J Clin Microbiol. 1997 Apr;35(4):907-14.

7 Supply P, Mazars E, Lesjean S, Vincent V, Gicquel B, Locht $C$. Variable human minisatellite-like regions in the Mycobacterium tuberculosis genome. Mol Microbiol. 2000 May;36(3):762-71.
8 Baver J, Andersen AB, Kremer K, Miorner H. Usefulness of Spoligotyping to discriminate IS6110 low-copynumber Mycobacterium tuberculosis complex strain cultured in Dennmark. J Clin Microbiol. 1999 Aug;37(8):2602-6.

9 Gouget de la Salmonière YO, Li HM, Torrea G, Bunschoten A, van Embden J, Gicquel B. Evaluation of Spoligotyping in a study of the transmission of Mycobacterium tuberculosis. J Clin Microbiol. 1997 Sep;35(9):2210-4.

10 Goyal M, Saunders NA, van Embden JD, Young DB, Shaw RJ. Differentiation of Mycobacterium tuberculosis isolates by spoligotyping and IS6110 Restriction Fragment Length Polymorphism. J Clin Microbiol. 1997 Mar;35(3):647-51.

11 Kremer K, van Soolingen D, Frothingham R, Haas WH, Hermans PWM, Martin C, et al. Comparison of methods based on different molecular epidemiological markers for typing of Mycobacterium tuberculosis strains: interlaboratory study of discriminatory power and reproducibility. J Clin Microbiol. 1999 Aug;37(8):2607-18.

12 Furlaneto IP, Sousa EB, Brito ML, Lima GLF, Lopes ML, Silva SHM, et al. Avaliação de diferentes procedimentos para extração de DNA a partir de esfregaços corados pelo método de Ziehl-Neelsen. Cad Saude Coletiva. 2007 jul-set;15(3):401 - 14.

13 Suresh N, Arora N, Pant H, Rana T, Singh UB. Spoligotyping of Mycobacterium tuberculosis DNA from archival Ziehl-Neelsen-stained sputum smears. J Microbiol Methods. 2007 Feb;68(2):291-5.

14 van der Zanden AGM, Hoentjen AH, Heilmann FG, Weltevreden EF, Schouls LM, van Embden JD. Simultaneous detection and strain differentiation of Mycobacterium tuberculosis complex in paraffin wax embedded tissues and in stained microscopic preparations. Mol Pathol. 1998 Aug;51 (4):209-14. 
15 Zink AR, Sola C, Reischl U, Grabner W, Rastogi N, Wolf $\mathrm{H}$, et al. Characterization of Mycobacterium tuberculosis Complex DNAs from Egyptian Mummies by Spoligotyping. J Clin Microbiol. 2003 Jan;41(1):359-67.

16 Parwati I, van Crevel R, van Soolingen D, van Der Zanden A. Application of Spoligotyping to noncultured Mycobacterium tuberculosis bacteria requires an optimized approach. J Clin Microbiol. 2003 Nov; 41 (1 1):5350-1

17 Vitol I, Driscoll J, Kurepina N, Kreiswirth B, Bennett K. SpotClust: a tool to cluster spoligotype data for tuberculosis evolution and epidemiology [Internet]. 2005 [cited 20 Feb 2010]. Available from: http:// ebookbrowse.com/8924918f4990ef1 fb3c31 a83e3 b8db 13-spotclust-recomb-pdf-d1 16432570

18 Ayres M, Ayres Júnior M, Ayres DL, Santos AS. BioEstat 5.0. Aplicações estatísticas nas áreas das Ciências Bio-Médicas. Belém: Instituto de Desenvolvimento Sustentável Mamirauá; 2007. 364 p.

19 Brudey K, Driscoll JR, Rigouts L, Prodinger WM, Gori A, Al-Hajoi SA, et al. Mycobacterium tuberculosis complex genetic diversity: mining the fourth international spoligotyping database (SpolDB4) for classification, population genetics and epidemiology. BMC Microbiol. 2006 Mar;6:23.

20 Scholante Silva AB, Von Groll A, Félix C, Conceição FR, Spies FS, Scaini CJ, et al. Clonal diversity of M. tuberculosis isolated in a sea port city in Brazil. Tuberculosis. 2009 Nov;89(6):443-7.

21 Gomes HM, Elias AR, Oelemann MA, Pereira MAS, Montes FFO, Marsico AG, et al. Spoligotypes of Mycobacterium tuberculosis complex isolates from patients residents of 11 states of Brazil. Infect Genet Evol. 2012 Jun; 12(4):649-56.

22 Basta PC, Oelemann MAC, Oelemann WMR, Fonseca LS, Coimbra Jr CEA. Detection of Mycobacterium tuberculosis in sputum from Suruí Indian subjects, Brazilian Amazon. Mem Inst Oswaldo Cruz. 2006 Sep; $101(6): 581-4$

23 Dalla Costa ER, Ribeiro MO, Silva MSN, Arnold LS, Rostirolla DC, Cafrune Pl, et al. Correlations of mutations in katG, oxyR-ahpC and inhA genes and in vitro susceptibility in Mycobacterium tuberculosis clinical strains segregated by spoligotype families from tuberculosis prevalent countries in South America. BMC Microbiol. 2009 Feb;9:39.

24 Guernier V, Sola C, Brudey K, Guégan JF, Rastogi N. Use of a cluster-graphs from Spoligotyping data to study genotype similarities and a comparison of three indices to quantify recent tuberculosis transmission among culture positive cases in French Guiana during a eight year period. BMC Infect Dis. 2008 Apr;8:46.

25 Candia N, Lopez B, Zozio T, Carrivale M, Diaz C, Russomando $G$, et al. First insight into Mycobacterium tuberculosis genetic diversity in Paraguay. BMC Microbiol. 2007 Aug; 7:75.
26 Sequera CM, Delgado SV, Araque MW, Torrealba OM, Núñez MR, da Mata JO, et al. Mycobacterium tuberculosis: espoligotipos en el Estado Carabobo, Venezuela. Rev Chil Infect. 2008 oct;25(5):362-7.

27 SITVIT. The SIVIT Database [Internet]. 2010 mar [cited 2010 mar 01] Available from: http://www.pasteurguadeloupe.fr:8081/SITVITDemo/

28 Aristimuño L, Armengol R, Cebollada A, España M, Guilarte A, Lafoz C, et al. Molecular characterization of Mycobacterium tuberculosis isolates in the first National Survey of Anti-tuberculosis drug resistance from Venezuela. BMC Microbiol. 2006 Oct;6:90.

29 Mancilla EM, Martínez HA, Palavecino BC, Rehren SG, Lucero LP, León RG, et al. Variantes genéticas de Mycobacterium tuberculosis aisladas de pacientes de la Xth Región de Chile. Rev Chilena Infectol. 2006 sep;23(3):220-5.

30 Malaspina AC, Cavalcanti HR, Leite CQF, Machado SMA, Viana BHJ, Silva RM, et al. Usefulness of Mycobacterium tuberculosis molecular typing in tuberculosis low-endemic agro-industrial setting of Brazil. Jpn J Infect Dis. 2008 May;61 (3):231-3.

31 Ritacco V, López B, Cafrune PI, Ferrazoli L, Suffys $\mathrm{PN}$, Candia N, et al. Mycobacterium tuberculosis strains of the Beijing genotype are rarely observed in tuberculosis patients in South America. Mem Inst Oswaldo Cruz. 2008 Aug;103(5):489-92.

32 Braden CR, Morlock GP, Woodley CL, Johnson KR, Colombel AC, Cave MD, et al. Simultaneous infection with multiple strains of Mycobacterium tuberculosis. Clin Infect Dis. 2001 Sep;33(6):42-7.

33 Richardson M, Carroll NM, Engelke E, Van Der Spuy GD, Salker F, Munch Z, et al. Multiple Mycobacterium tuberculosis strains in early cultures from patients in a high-incidence community setting. J Clin Microbiol. 2002 Aug;40(8):2750-4

34 Shamputa IC, Jugheli L, Sadradze N, Willery E, Portaels F, Suplly P, et al. Mixed infection and clonal representativeness of a single sputum sample in tuberculosis patients from a penitentiary hospital in Georgia. Respir Res. 2006 Jul;7:99.

35 Stavrum R, Mphahlele M, Ovreas K, Muthivhi T, Fourie PB, Weyer K, et al. High diversity of Mycobacterium tuberculosis genotypes in South Africa and preponderance of mixed infections among ST53 isolates. J Clin Microbiol. 2009 Jun;47(6): 1848-56.

36 Warren RM, Victor TC, Streicher EM, Richardson M, Beyer SN, van Pittius NCG, et al. Patients with active tuberculosis often have different strains in the same sputum specimen. Am J Respir Crit Care Med. 2004 Mar;169(5):610-4.

37 Longo MC, Berninger MS, Hartley JL. Use of uracil DNA glicosylase to control carry-over conatmination in polymerase chain reactions. Gene. 1990 Sep; 93(1):125-8. 
38 Assunção RM, Barreto SM, Guerra HL, Sakurai E. Mapas de taxas epidemiológicas: uma abordagem Bayesiana. Cad Saude Publica. 1998 outdez; 14(4):713-23.

39 Instituto Brasileiro de Geografia e Estatística. Banco de Dados Agregados. SIDRA: Sistema IBGE de Recuperação Automática. Tabela 156. Média de moradores por domicílio [Internet]. 2010 [citado 2012 mar 12]. Disponível em: http://www.sidra.ibge.gov.br/

40 Instituto Brasileiro de Geografia e Estatística. Banco de Dados Agregados. SIDRA: Sistema IBGE de Recuperação Automática. Tabela 202. População Residente por sexo e situação de domicílio [Internet]. 2012 [citado 2012 mar 12]. Disponível em: http:// www.sidra.ibge.gov.br/
41 Santos CB, Hino P, Cunha TN, Villa TCS, Muniz JN. Utilização de um Sistema de Informação Geográfica para descrição dos casos de tuberculose. Bol Pneumol Sanit. $2004 ; 12(1): 5-10$.

42 Cafrune PI, Possuelo LG, Riberio AW, Ribeiro MO, Unis G, Jarczewski CA, et al. Prospective study applying Spoligotyping directly to DNA from sputum samples of patients suspected of having tuberculosis. Can J Microbiol. 2009 Jul;55(7):895-900.

43 Hayward AC, Watson JM. Typing mycobacteria using Spoligotyping. Thorax. 1998 May;53(5):329-30.

44 Kato-Maeda M, Small PM. How molecular epidemiology has changed what we know about tuberculosis. West J Med. 2000 Apr;172(4):256-9. 